\title{
Experimental study of two-dimensional turbulence in square domains
}

\author{
Ding Long \\ School of Mechanical, Shanghai Institute of Technology, Shanghai 201418, China \\ dlong2014@126.com
}

Keywords: electromagnetically forced 2-D turbulence; PIV; double scaling law.

\begin{abstract}
We built the square structure model of two-dimensional turbulence experiments, using of PIV measured velocity field and the vortices field of the two-dimensional turbulent and analyzed to obtain an energy spectrum, you can clearly see the double-scaling law characteristic in the energy spectrum, and the turning point of the two-scale cascade area happens to be the energy injected into the experimental system scales.
\end{abstract}

\section{Introduction}

Unpredictability is an essential property of turbulent flows. Turbulence is characterized by a large number of degrees of freedom interacting with nonlinear dynamics. Initially, the study of 2-D turbulence is a simplified model of the traditional turbulence, however, with the progress of research found the problem of two-dimensional turbulence is far from simple one-dimensional reduction [1], in a sense, it is a question of a new non-linear systems [2].

Although at small scales flows in the atmosphere and oceans are undeniably 3D, their lateral extent is so much larger than their depth that large-scale motion lies primarily in the plane. Recent studies of atmospheric have also confirmed that with increasing of space scale, the characteristics of atmospheric turbulence transformed into the two-dimensional turbulence [3].Some scholars believe studying ideal 2-D turbulence can deepen the understanding of turbulence [4]. Currently, research on 2-D turbulence mainly about the evolution of fully developed turbulence in the infinite domain or square, round closed domain. In 1986, Sommeria first conducted research of 2-D turbulence [5].Professor G.A. Voth of Wesleyan University had studied the particle dynamics in 2-D turbulence [6], and improved the experimental techniques and analysis methods [7]. Professor N. T. Ouellette of Yale University researched the machine problem by experimental study [8-10].

\section{Experiment}

We study 2D-turbulence in an electromagnetically driven fluid layer as shown in Fig. 1.A electric current travels horizontally through a fluid layer that is placed above an array of permanent magnets. The resulting Lorentz forces drive a vortex array flow in the fluid. Used plexiglass tank wall to ensure that the insulation resistance of the device where the solution is energized, and translucent when collecting data and shooting. Tank is rectangular in shape, the size of the inner wall is $300 \times 330 \mathrm{~mm}^{2}$,the experimental area is $300 \times 300 \mathrm{~mm}^{2}$.The electrolyte sits on top of a glass bottom plate; beneath the glass is a square $11 \times 11$ grid of neodymium-iron-boron $(\mathrm{NdFeB})$ grade $\mathrm{N} 35$ magnets, spaced $20 \mathrm{~mm}$ on center. Each magnet is cylindrical, with a diameter of $10 \mathrm{~mm}$, a thickness of $5 \mathrm{~mm}$. The working fluid is a solution of $20 \%$ by mass $\mathrm{NaCl}$ in water. The Reynolds number is defined as $\mathrm{Re}=\mathrm{UL} / v \square$, where $\mathrm{U}$ is the root mean square fluid velocity, $\mathrm{L}$ is the forcing length scale given by the typical magnet spacing, and $v \square$ is the kinematic viscosity of the fluid.

Using PIV to get the information of flow field. We seed the flow with small fluorescent particles (Pollen).With a specific gravity of 1.21, these tracer float at the interface between the salty layer and the pure water layer; since this interface does not have a bulk surface tension, there are no long-range forces coupling the particles. 


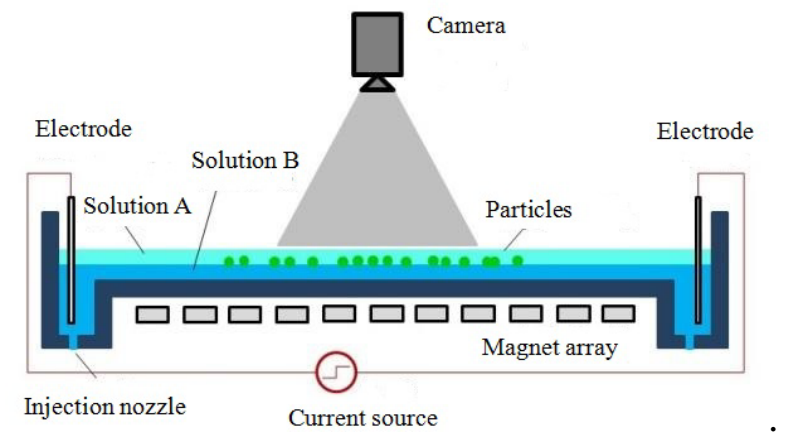

a

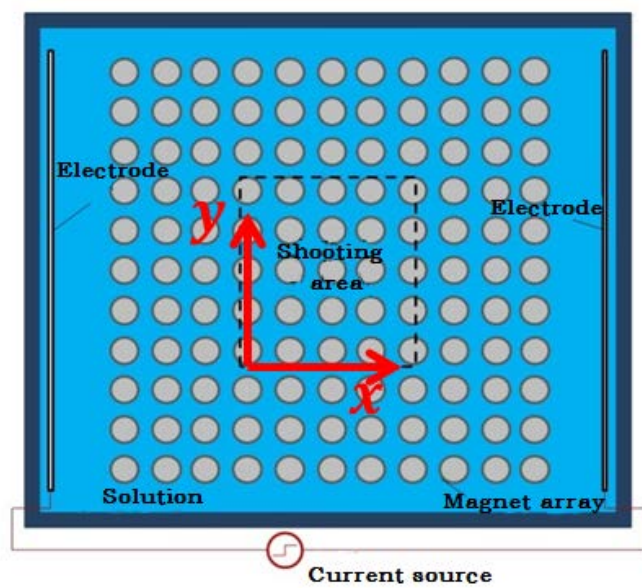

$\mathrm{b}$

Fig.1 Schematic of data acquisition

\section{Results and discussion}

Tab.1 Parameters of PIV experiments

\begin{tabular}{ccc}
\hline Numble & Name & Value \\
\hline 1 & Temperature & $25^{\circ} \mathrm{C}$ \\
2 & The experimental solution & single salt solution \\
4 & Thickness & $4 \mathrm{~mm}$ \\
5 & Dynamic viscosity & $1.271 \times 10^{-3} \mathrm{~Pa} \cdot \mathrm{s}$ \\
6 & Characteristic scale & $20 \mathrm{~mm}$ \\
7 & Reynolds & 98.2 \\
8 & Picture pixels & $1024 \times 1024$ \\
9 & frequency & $250 \mathrm{~Hz}$ \\
10 & Kolmogorov scale & $200 \mu \mathrm{m}$ \\
\hline
\end{tabular}

Table 1 lists the detailed parameters. The distance between the axes of adjacent magnets is defined as the characteristic scale, that is also the injection scale of system energy $l_{f}=1 / k_{f}$ ( $k_{f}$ is the wave number corresponding to the injection scale).

We use Prana software processing the picture data to get velocity field information. Figure 2 (a) shows the velocity field at a certain time $t_{*}$ after reaching steady state, color indicates the magnitude of speed.(b) shows the vorticity field at time $t_{*}$, color indicates the magnitude of vortices strength. Comparing Figure 3 (a) and (b), can be found in the region of speed changing intense has a larger magnitude in the vorticity field, which is more intense vortex motion. We also processing the data, 
obtained energy spectrum of two-dimensional turbulent velocity field (Figure 3), you can see two cascade area existing on both sides of the energy injection scale $\left(l_{f}=1 / k_{f} \approx 20 \mathrm{~mm}\right)$, the dotted line is the predicted value of K67 theory for two-dimensional turbulent cascade area scaling law.

Can be seen from the figure, the experimental data is consistent with the theoretical prediction of K67, but not identical. The main reason leading to the deviation that the existence of Ekman friction in two-dimensional electromagnetically driven fluid flows, while K67 theoretical analysis did not consider the role of Ekman friction. Therefore, in subsequent studies research group will focus on the impact of Ekman friction to the two-dimensional turbulence scale.
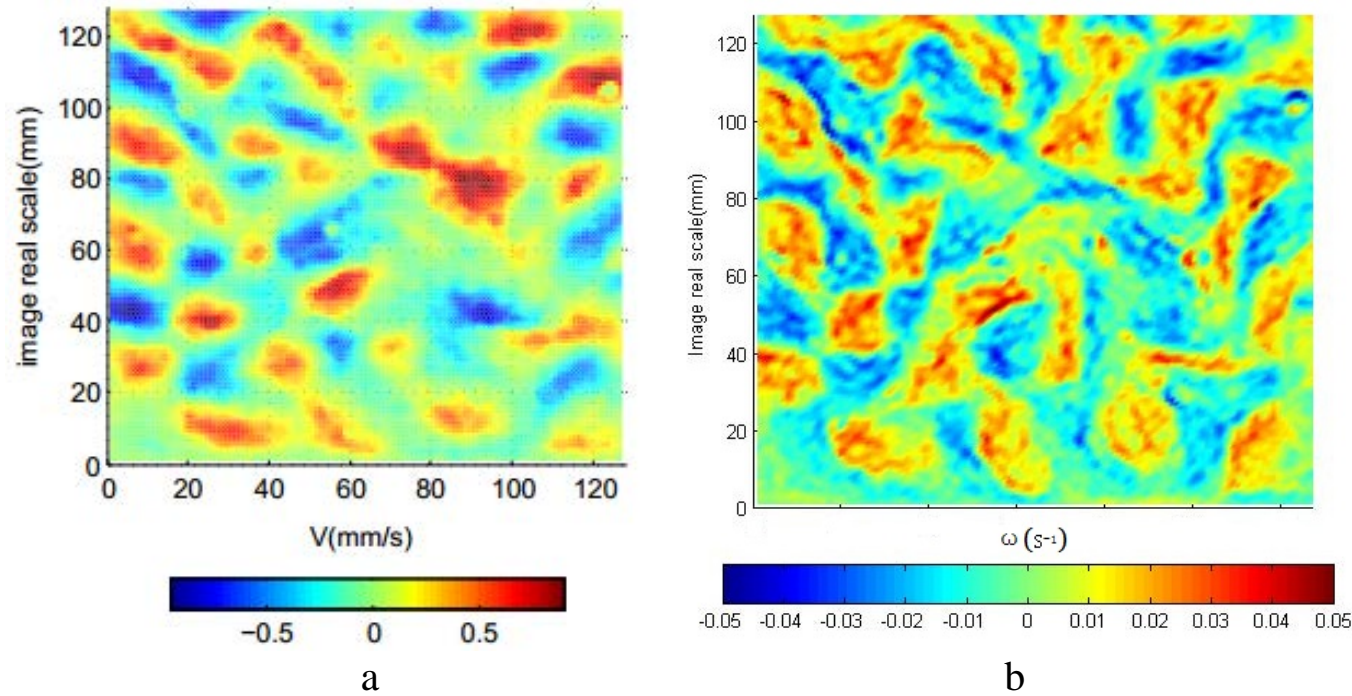

Fig.2 Velocity and vorticity field.

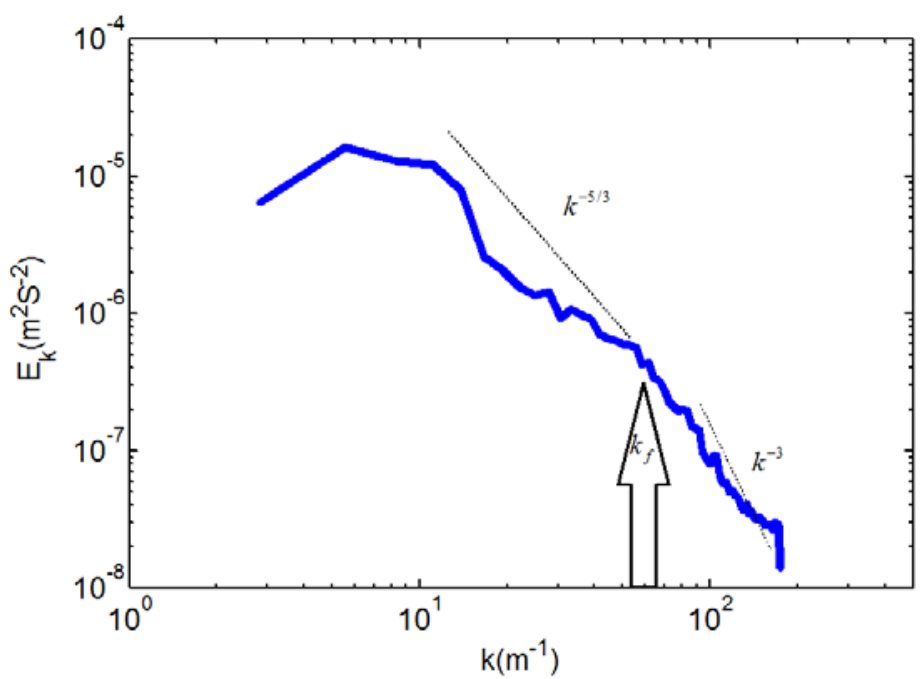

Fig.3 Energy spectrum of velocity field

The influence of the underlying forcing geometry is apparent in an ensemble average of flow fields in Figure 4 (a), color indicates vorticity. From the figure can be clearly seen vortices array and magnitude of positive and negative vorticity staggered. Enlarging a local region in (a), and drawing the vector fields corresponding to the time, as shown in Figure 4 (b), can be found the array of vortex result in an array of vorticity. Here can be found that when in laminar flow state (the Lorentz force is small), the distribution of the vortex array corresponds to the distribution of the magnet array. This phenomenon can be summarized as follows: In laminar flow, vortex array is corresponding to the magnet array; in two-dimensional turbulent, the vortex array in the time-averaged flow field and the magnet array are inconsistent. This phenomenon still needs further experimental studies. 

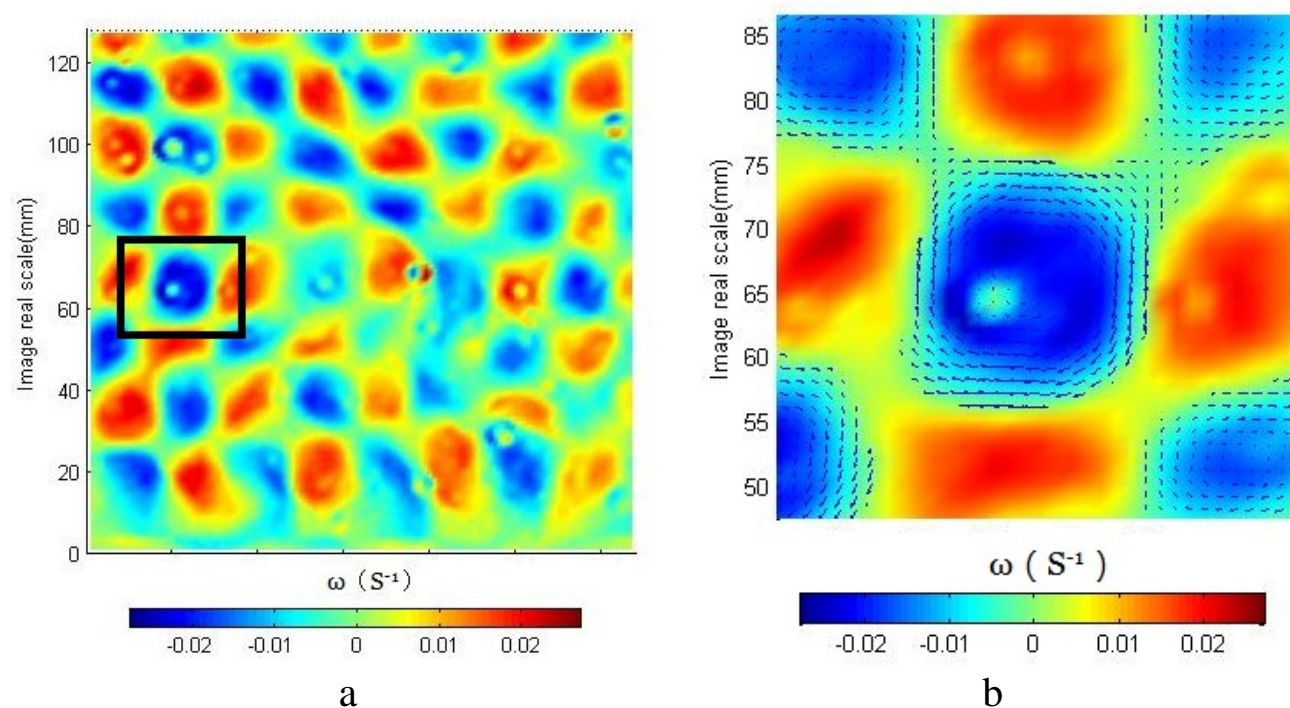

Fig.4 Vorticity field.(a) Vorticity field of time averaging.(b)The enlarged view.

\section{Summary}

We obtain the velocity field and vorticity field of two-dimensional turbulence by PIV.The energy spectrum can be obtained by analyzing the velocity field, which has the double-scaling law characteristic, and the turning point of the two-scale cascade area happens to be the energy injected into the experimental system scales, however, due to the existence of Ekman friction led to the scaling exponent of two cascade area is not consistent with the K67 theory.

\section{References}

[1] G. Boffetta,R.E.Ecke.Two-dimensional turbulence[J].Annual Review of Fluid Mechanics, 2012, 44: 427-451.

[2] N.T. Ouellette.Turbulence in two dimensions[J]. Physics Today, 2012, 65(5): 68.

[3] R.Ni,N.T.Ouellette,G.A.Voth.Alignment of vorticity and rods with Lagrangian fluid stretching in turbulence [J].arXiv preprint arXiv: 1311.0739, 2013.

[4] S.B.Kuksin,A.Shirikyan.Mathematics of two-dimensional turbulence[M].Cambridge University Press Cambridge, 2012: 35-94

[5] A.Adamczyk, L. Rimai. 2-Dimensional particle tracking velocimetry (PTV): technique and image processing algorithms [J]. Experiments in fluids, 1988, 6(6): 373-380.

[6] S.Parsa,J.S.Guasto,M.Kishore,N.T.Ouellette.Rotation and alignment of rods in two-dimensional chaotic flow[J]. Physics of Fluids, 2011, 23: 043302.

[7] R.Ni,N.T. Ouellette,G.A.Voth. Alignment of vorticity and rods with Lagrangian fluid stretching in turbulence[J].arXiv preprint arXiv: 1311.0739, 2013.

[8] N.T.Ouellette,P. O’Malley, J. P. Gollub. Transport of finite-sized particles in chaotic flow[J]. Physical review letters, 2008, 101(17): 174504.

[9] Y. Liao,N.T.Ouellette. Spatial structure of spectral transport in two-dimensional flow[J]. Journal of Fluid Mechanics, 2013, 725: 281-298.

[10] Y.Liao,D.H.Kelley,N.T.Ouellette. Effects of forcing geometry on two-dimensional weak turbulence[J].Physical Review E,2012,86(3):036306. 